\title{
CHARISMA-KORRELATE IN SPRACHE UND DISKURS: KANN EIN IDEALTYP EIN OBJEKT DER EXPERIMENTAL-PHONETISCHEN FORSCHUNG SEIN?
}

\author{
Natalja Petljutschenko ${ }^{1}$
}

\begin{abstract}
The paper presents an analysis of charisma-correlates in the language of political leaders in modern German and Ukrainian political discourse and answers the question of whether an ideal type can be an object of multimodal phonetical research. The discursive description of charismatic leaders in German and Ukrainian political discourse and the discovery of their contrastive features relies on biological, social, psychological and linguistic parameters forming their charismatic discourse portraits. Of decisive importance in this context, is the rhetoric of public appearance, expressive combative position, hortatority, timbral, prosodic and gesture-mimic characteristics further perceived and attributed to its carriers as charisma. Charismatic politicians have always been characterised by sharp statements, categorical views and mobilising speech. Inspirited or pep rhetoric is inherent in politicians whose position is contrary to the majority of the public, the opinion of their political allies, members of Parliament, etc. We can observe its manifestations in moments of acute crisis in political life. The prosodic specifics of charismatical discourse are characterised by intensification of all its dynamic, tonal, and temporal components. In phonetical studies, this acoustic effect is referred to as prosodic intensity understanding it as abrupt changes in pitch, loudness, tempo variations, and pauses in important utterances containing addresses, appeals and concepts with positive semantics. Charismatic rhetoric is also formed by the kinetic (gesture and mimic) component that is functionally related the prosodic representation of speech making communication more effective. A gesture is the action or movement of the body through which one individual signals another individual about his presence, his intentions regarding objects. Charismatic political communications are characterised primarily by accentuating or co-speech gestures that represent movements of the body, especially the arms/hands, by which the speaker explains, complements his words, highlights the key points, emphasises or amplifies a verbal utterance. A gesture is perceived by the addressee as a kinematic form of verbal charisma-appeal through which he exercises his influence on his followers and/or opponents encouraging them to commit actions aimed at achieving a particular goal.

Charismatic rhetoric originates in political communication in times of crisis and is characteristic of politicians whose stand is not consistent with the majority position. Charismatic enthusiasm and inspiration are ethnically coloured (German rapsodicity, Ukrainian monotony) and are reflected in prosodic and gesture emphasis accompanying both independent and dependent parts of speech indicating the autonomy of charismatic rhetoric as a model. The results of this study can be applied in discourse studies, applied phonetics, comparative linguistics, and political communication.
\end{abstract}

Keywords

Ideal type, charisma, prosodic highlighting, enthusiasm, inspiration, rapsodicity, monotony, co-speech gestures, mimics.

1. Einführung. Im deutschen und ukrainischen politischen Diskurs zeigt sich momentan ein steigendes Interesse an dem Charisma-Konzept des berühmten deutschen Soziologen Max Weber ${ }^{2}$ und im Ergebnis - werden die Sehnsuchtsrufe nach neuen politischen Persönlichkeiten mit Funkenflug, die sich von großen Ideen und Werten inspirieren lassen und damit auch andere Menschen «anstecken», laut. Die Besonderheit der Situation mit den Charismatikern im modernen Deutschland besteht jedoch darin, dass sich aktuell in der Heimat des Konzepts ein "Charisma-Vakuum» gebildet hat, das nicht nur auf die langanhaltende rationale Herrschaft von Angela Merkel zurückzuführen ist ${ }^{3}$, sondern auch auf den

\footnotetext{
1 Natalia Petlyuchenko, National University Odessa Law Academy, Fontanskaya Doroga, 23, 65009-Odessa, Ukraine, Email: natalja.petljutschenko@onua.edu.ua. ORCID ID: 0000-0002-8089-2947

${ }^{2}$ Weber 2006, Schwinn 2016

${ }^{3}$ Dempsey 2013

${ }^{4}$ Encke 2014, Haese 2017

${ }^{5}$ Schmölders 2000, Herbst 2011

${ }^{6}$ Langguth 2009

${ }^{7}$ Gössler 2009
}

Mangel an charismatischen Krisenmanagern in der heutigen schwierigen Situation mit den Flüchtlingen, die zusammen mit den für sie und gegen sie kämpfenden Links- und Rechtsradikalen die Landschaft der deutschen Straßen und die deutsche Politiklandschaft stark verändert haben.

Gleichzeitig stellt sich häufig aber die Frage, ob deutsche Politiker überhaupt ein Charisma brauchen ${ }^{4}$, welches vor dem historischen Hintergrund der Deutschen bereits negative Konnotationen besitzt und sich primär auf Nationalsozialismus und vor allem auf das «dunkle» Charisma Hitlers bezieht ${ }^{5}$. Eben aus diesem Grund war das Charisma-Konzept lange Zeit aus dem positiven Arsenal rhetorischer und argumentativer Strategien eines deutschen Politikers und vor allem des Kanzlers/der Kanzlerin strikt ausgeschlossen ${ }^{6}$. Beim besten Willen der Deutschen, einen «frischen», leidenschaftlichen Charismatiker wie Emmanuel Macron (Frankreich) oder Barack Obama (USA) zu ergattern ${ }^{7}$, und sogar trotz der Vielzahl von schillernden Persönlichkeiten mit Charisma wie Sarah Wagenknecht, Christian Lindner, Gregor Gysi, Robert Habeck, Annalena Baerbok u.a.m., gibt es einen solchen Influencer im heutigen Deutschland nicht. Die Bereitschaft zu dessen Projektion ist 
jedoch aber groß, besonders nach dem überraschenden Erfolg der rechtpopulistischen «Alternative für Deutschland» (AfD) bei den Landtagswahlen in Bayern und Hessen.

In der Ukraine, die sich derzeit in einem nicht deklarierten Kriegszustand befindet und zugleich am Vorabend der neuen Präsidentschafts- und Parlamentswahlen steht, ist der Bedarf nach einem neuen charismatischen Führer ebenfalls ziemlich groß. In diesem Kontext muss betont werden, dass der charismatische Führungstyp, der sich in der Ukraine erstmals in einer konkreten/klaren Form im Ersten Maidan («Orangene Revolution») bemerkbar machte und im Westen mit den Namen von W. Juschtschenko und J. Timoschenko verbunden war, in der Zeit nach dem Zweiten Maidan/Euromaidan ( «Revolution der Würde») eine Wandlung erfahren hat und sich durch «Decharismatisierung» des politischen ukrainischen Establishments gekennzeichnet ${ }^{8}$. Die Nachfrage nach einem mobilisierenden Charisma ist gesunken: denn Leidenschaft und Inspiration werden überwiegend während Revolutionen benötigt, für den Aufbau der Wirtschaft und die Einstellung der militärischen Handlungen im Osten der Ukraine wären jedoch rationale Argumente und Stabilität von großer Bedeutung.

Im vorliegenden Beitrag möchte ich mich auf vier wichtige Themen konzentrieren, und zwar 1) Charisma als Idealtyp\& Konzept, 2) Charismas als ein psychoemotioneller Zustand der Begeisterung, 3) kontrastive sprachliche und parasprachliche Charisma-Korrelate in den Auftritten von mobilisierenden Charisma-Trägern - Joschka Fischer und Julia Timoschenko (Deutsch/Ukrainisch).

2. Zum Forschungsproblem. Die bisherigen Untersuchungen des Charisma-Phänomens gehörten $\mathrm{zu}$ den Forschungsgebieten solcher Wissenschaften wie Theologie, Soziologie, Philosophie, Psychologie, Politologie, Anthropologie und PR-Technologien, die ein breites Spektrum an Charisma-Definitionen von der Gottesgnade (von griech. $\chi \dot{\alpha} \rho l \sigma \mu \alpha$ - Gabe, Geschenk) bis zu der Ausstrahlung, Anziehungskraft (vom franz. Charme, Flair flev), Fluidität, Aura usw. aufweisen. Bisher wurde allerdings eine offene Tatsache außer Acht gelassen, dass jeder Politiker - auch ein charismatischer - homo loquens ist, der seinen Einfluss auf Nachfolger und Gegner erstmal mithilfe von Worten (auch von Gesten) ausübt und deswegen in die Forschungszonen der Linguistik, Linguopragmatik, Linguokulturologie und interkulturellen Kommunikation eingeschlossen werden kann.

Heutzutage kann man in den Recherchen bis zu den 60 Definitionen von Charisma finden, die meisten von denen sich auf Max Weber beziehen, der den Begriff «Charisma» in der Soziologie nutzte, um eine

\footnotetext{
${ }^{8}$ Petljutschenko 2014

${ }^{9}$ Petliuchenko 2009
}

der drei von ihm unterschiedenen Formen der Herrschaft zu bezeichnen - neben «traditionaler» und «rationaler» Herrschaft - eine charismatische Herrschaft. Im Anschluss an Weber bezeichnet Charisma eine soziale Beziehung von Herrschaft, welche die Sozialstruktur grundlegend verändert, eine außeralltägliche «revolutionäre Macht», und zwar so, wie sie von den charismatisch Beherrschten, den Anhängern («Jüngern»), gewertet wird - also es handelt sich im Prinzip in diesem Fall - nach Weber - um eine Zuschreibung. Diese Anerkennung, meint Weber, ist psychologisch eine aus Begeisterung und Vertrauen geborene ganz persönliche Hingabe.

Die durchgeführte Untersuchung hatte das Ziel, einen Beitrag zu einer linguistischen Interpretation des Weberschen Charisma-Idealtyps zu leisten und darüber hinaus ein experimentelles Sensorium zu entwickeln, «um ein Charisma nach äußeren Indizien empirisch nachzuweisen». In der Monographie «Charyzmatyka : movna osobystist' i dyskurs» ${ }^{9}$ wird zum ersten Mal folgende linguistische Interpretation des Charisma-Phänomens am Beispiel des politischen Diskurses vorgeschlagen: zunächst wird Charisma als ein interdisziplinäres Phänomen betrachtet, danach wird sein diskursiver Status im Rahmen der sprachlichen charismatischen Persönlichkeit des politischen Führers - also homo charismaticus beschrieben, und dann werden Besonderheiten der charismatischen Appellativität des politischen Führers in der deutschen und ukrainischen Sprachkulturen mithilfe einer kontrastiven Analyse ermittelt.

Ausgehend von und im Hinblick auf M. Weber wird zugrunde gelegt, dass der charismatische Führer in einer Krisenzeit, also bei großen sozialen Wandlungen in der Gesellschaft und Revolutionen auf jegliche Art rationeller Einstellungen in seinen Handlungen verzichtet und einen emotionellen verbalen Einfluss ausübt, dessen Grundlage der psychoemotionelle Zustand der starken Begeisterung, des inneren Triebs ausmacht.

Der Maßstab, die Bedeutung der historischen Situation, an der der Charismatiker selbst teilnimmt, die großen Ideen wie Freiheit, Unabhängigkeit, Zukunft, Einheit usw. bewegen ihn. In diesem Zustand passiert mit ihm das, wovon Max Weber geschrieben hat und was er selber einmal erlebt hat - das so genannte «Aus-sich-Heraustreten», die Ekstase. Der Politiker wird von großen Ideen begeistert, wird davon mitgerissen und reißt durch diese Ideen die anderen mit. Und wenn sie mich fragen, was ist in Ihrem Verständnis Charisma, welcher Politiker als charismatisch zu bewerten wäre, so antworte ich: Charisma ist die Fähigkeit, sich selbst von großen Ideen begeistern zu können und dann die anderen dadurch mitzureißen! In der Krise steigt die Zahl der in den politischen Auftritten von den Politikern verwendeten Appelle. Die «Krisen-Auftritte» charakterisieren sich durch einen hohen Anteil der kommunikativen Sprechakte 
«Aufforderung». So kommen wir zu einer Annahme, also zu einer Hypothese über der appellativen Dominante im Diskurs des charismatischen politischen Führers. Die Graduierung der Appellativität - stark, mäßig und schwach bestimmt dementsprechend den Typ des Charismatikers - einen genuinen, situativen und schwachen bis zu einem inszenierten. Die vorgeschlagene Vorgehensweise erlaubt es uns, bei der Untersuchung des Phänomens des politischen Charismas einerseits von der Ebene der immateriellen, unverständlichen, übernatürlichen Eigenschaft eines Menschen (ob angeboren oder angeeignet) in die Ebene des messbaren emotionalen Zustandes zu wechseln, anderseits ein linguistisches Äquivalent Appellativität in seinem Diskurs zu finden.

\section{Ergebnisse und Diskussion.}

3.1. Diskursive Kontraste. Diskursive Kontraste bei der Zuschreibung der charismatischen Eigenschaften den Politikern in den deutschen und ukrainischen Sprachkulturen zeigen sich vor allem in der verschiedenen Gestalt-Wahrnehmung/Erwartung des Führers von jedem Volk. So orientieren sich die Zuschreibungscharakteristika (rus. mifologemy) deutscher Charismatiker vor allem an der Größe einer konkreten historischen Situation und an jener Rolle, die der jeweilige Politiker darin spielte.

Durch solche Erwartungen werden folgende Zuschreibungen bestimmt wie «Verkörperung der Nation, des nationalen Gedankens selbst», «Identität mit Deutschland», «der nobelste Kerl des Erdkreises» - für Heinrich von Gagern, «eiserner Kanzler», «der Baumeister des Reiches» - für Otto von Bismarck, «Beruhigungsfaktor für Deutsche in den Nachkriegsjahren», «Ein Denkmal seiner Zeit»für Konrad Adenauer, «deutscher Kennedy» - für Willi Brandt, «Kanzler der Einheit» - für Helmut Kohl. Bei den modernen Politikern sind die Zuschreibungsklischees anders: «Medienkanzler» - für Gerhard Schröder, «Turnschuh-Minister», «Chamäleon» - für Joschka Fischer, «Mutti», «Mutter der Nation» - für Angela Merkel ${ }^{10}$.

Die Zuschreibungsklischees für ukrainische Charismatiker spiegeln die Erwartungen eines legendären Helden, eines Kämpfers für die nationale ukrainische Idee wider. Durch solche Erwartungen werden folgende Zuschreibungen bestimmt wie «Held der ukrainischen Nation», «der Grosse Hetman», «Verräter des ukrainischen Volkes» - für Bohdan Hmelnyzkyj, «Held der Nation», «Verräter des ukrainischen Volkes» - für Ivan Mazepa, (Sie sehen, die Zuschreibungen für Bohdan Hmelnyzkyj und Ivan Mazepa widersprüchlich sind, sie wurden aus verschiedenen Perspektiven gemacht) «legendäre Persönlichkeit», «Revolutionär-Rebell» - für Nestor Machno, «tap-

\footnotetext{
${ }^{10}$ Petliuchenko 2009, pp. 435-447

${ }^{11}$ ibid, pp. 448-454
}

ferer Kämpfer für eine unabhängige Republik» - für Simon Petljura, «angeborener Führer», «Verkörperung der ukrainischen Nationalidee» - für Vjacheslav Chernovil. Bei den modernen finden wir solche wie «Symbol der Orangenen Revolution» - für Wiktor Juschtschenko, «Jila», «Engel der Ukraine», «Königin des Maidans», «Evita von Kiew» - für Julia Timoschenko ${ }^{11}$.

3.2. Verbale Kontraste. Sprachliche Kontraste in den charismatischen Diskursen ukrainischer und deutscher Politiker zeigen sich vor allem in verschieden pragmatischen Einstellungen (Motivationen) bei den kommunikativen Taktiken des Appellierens.

Kontrastive Merkmale der Appellativität des deutschen charismatischen Diskurses sind die Aufforderungen, die pragmatisch, rational gefärbt sind 1) das gemeinsame Ziel zu finden, das den Führer und seine Nachfolger vereinigt, 2) Zukunftsperspektiven zu bestimmen sowie 4) so schnell wie möglich Fragen der Zeit zu lösen.

Kontrastive Merkmale der Appellativität des ukrainischen charismatischen Diskurses explizieren die Appelle, die sich auf Irrationelles der Nachfolger orientieren, und zwar 1) an die besondere Mission der ukrainischen Nation, 2) an den orthodoxen Glauben der Ukrainer, und 3) an Gott, zum B.: (Wiktor Juschtschenko: Wie ihr auch, bete ich diese Tage zu Gott um die Ukraine. Ich bitte ihn, uns alle mit Weisheit und Stärke zu beschenken, damit wir dem Unrecht, das die jetzige regierung verbreitet, widerstehen können.Sie unterstehen einem (einzigen, gemeinsamen) Gebot: Gott bewahre dich, dass du dich mit deinen Nachbarn streitest!Як і ви, у ці дні я молю Бога за Україну. Я прошу Його послати нам усім мудрість і силу, щоб протистояти неправді, яку сіє між нас нинішня влада; Ви підкоряєтесь одній заповіді: боронь Боже, щоб ти з сусідами лаявся!). Es wird vermutet, dass der Charismatiker direkt an den Gott appellieren und von Ihm die Gabe der Prophezeiung bekommen kann. So ist die Appellation der ukrainischen Führungspersonen durch zahlreiche Prophezeiungen, Weissagungen gefärbt, z.B.: ich prophezeie, ich sehe vorher, ich sage vorher, ich sehe und verkünde die Zukunft я пророкую, я завбачаю, я провіщаю, я бачу й оголошую наперед майбутнє usw.

Der deutsche Politiker gebraucht stattdessen solche Performative wie ich weiss, ich glaube, ich verstehe, ich sehe, ich bin in der Lage usw. Zudem fehlt im deutschen charismatischen Diskurs die Tonalität der Prophezeiung der besonderen Mission Deutschlands auch deswegen, weil sie negative Assoziationen in der Geschichte zum besonderen Deutschen Weg hervorrufen kann und deutsche Politiker berücksichtigen diesen Faktor in ihren appellativen Taktiken. Sie formulieren Ihre Zukunftsvisionen, was Deutschland angeht, eher in Form der Aussichten als Prophezeiungen. 
3.3. Parasprachliche Kontraste. Die Realisierung von prosodischen und kinetischen Mitteln der Gestaltung von appellativen Einheiten in der begeisterten Rede von charismatischen politischen Anführern wird im Rahmen der so genannten appellativen Welle betrachtet, unter welcher eine bestimmte Verteilung von Aufforderungen, also Appellen im Laufe eines gesamten Auftritts verstanden wird. Diese begeisterten Aufforderungen gestalten den charismatischen Spielraum des Sprechers und kennzeichnen sich durch Höhepunkte und Absenkungen von akustischen Parametern wie Dauer, Tonhöhe und Intensität, was den akustischen Effekt einer wellenartigen prosodischen Intensivierung von dem Beginn bis zum Ende des Auftritts bewirkt.

Die Prosodie, die sich eine große Amplitude aller akustischen Parameter des Sprachsignals vom Beginn der Welle bis zu ihrem Finale (d.h. vom Beginn des Auftritts bis zu seinem Finale) aufweist, heißt rhapsodisch (engl. rhapsodical - exaltiert, rhapsodical speech - begeisterte Rede), charakterisiert sich durch einen langsamen und stillen (leisen) Einstieg in die Rede mit einem abrupten Wechsel von Tempo und Lautstärke, die an den Höhepunkten der Rede einen maximalen Grad erreichen). Eben diese Art und Weise der prosodischen Gestaltung der Rede gibt den begeisterten Zustand wieder. Wenn die Amplitude der akustischen Parameter minimal ist oder fehlt, so heißt eine solche Prosodie nach monoton $^{12}$.

Kinetische charismatische Spezifik zeichnet sich durch gestische Intensivierung an den Höhepunkten der Welle, und zwar durch akzentuierende Co-Speech-Gesten meistens der rechten Hand (bekannt wie «Römischer Gruß»), welche nach Kendon als redebegleitende Gesten des charismatischen politischen Führers bezeichnet werden. In der Kinematik haben wir gemeinsame Merkmale für Politiker von beiden Sprachkulturen festgestellt wie z.B. Lage der Hand an den Kulminationsstellen des Appells über der Schulterlinie, die Form der Hand - Faust, offene Handfläche oder Faust mit gestrecktem Zeigefinger, Richtung der Bewegung der Hand - zum Publikum und nach oben, Charakter der Bewegung - impulsiv.

Als Analyse-Beispiel des rhapsodischen Aufbaus der Prosodie möchte ich einen Ausschnitt aus der Rede von Joschka Fischer bei der Parteikonferenz zum Nato-Einsatz im Kosovo (13 Mai 1999, Bielefeld ${ }^{13}$ ) anführen, wo sein Auftritt als einer der stärksten in Bezug auf politische Rhetorik anerkannt wurde und wo Fischer seine charismatische Kraft am stärksten zum Ausdruck gebracht hat. Auf seiner Suche nach einer ehrlichen und offenen Diskussion mit dem Publikum und in seinem Streben, bei den scharfen Fragen an eine Verständigung zu gelangen, benutzt

\footnotetext{
${ }^{12}$ Petlyuchenko \& Artiukhova 2015

${ }^{13}$ Fischer 1999
}

Fischer die Taktik des allgemeinen impliziten Appells in Form von Fragesätzen und wendet dabei die Technik der Fragenkaskade an: [1] Was ich mich frage ist [Pfeifen]... [2] was ich mich frage ist [Pfeifen] [3] warum ihr diese Diskussion verweigert? [Pfeifen] [4]. Warum verweigert ihr mit Trillerpfeifen diese Diskussion, [5] wenn ihr euch als Linke oder gar Linksradikale bezeichnet? [6]Ihr mögt ja alles falsch finden, was diese Bundesregierung gemacht hat und die Nato macht [7] das mögt ihr alles falsch finden! [Pfeifen, stürmischer Beifall].

Wie aus dem angeführten Beispiel ersichtlich ist (Syntagmen 1-3), stellt er Fragen quasi an sich selber (was ich mich frage ist, warum ihr diese Diskussion verweigert?). Er macht das in einer heruntergesetzten Grundfrequenz- und Intensitätslage, unter Verwendung der Methode des so genannten langsamen, also verzögerten Starts. Danach wendet sich Fischer mit derselben Frage (Warum verweigert ihr mit Trillerpfeifen diese Diskussion, wenn ihr euch als Linke oder gar Linksradikale bezeichnet?) direkt an die Zuhörer, wobei er die Grundfrequenz- und Intensitätslage verändert und auf den Effekt der temporalen Zeitraffung (rus.temporalnoe sshatie), also Kompression zurückgreift.

So verringert sich die Dauer von Pausen vom Anfang einer Welle bis zu ihrem Höhepunkt im Vergleich praktisch um das fünffache: von $3500 \mathrm{Mc}$ - die erste Pause, $1500 \mathrm{mc}$ - die zweite Pause, 1200 мc die dritte, $720 \mathrm{mc}$ - die vierte, $600 \mathrm{mc}$ - die fünfte, danach verschmilzt alles in einem ununterbrochenen pausenlosen Redestrom, welcher in einer $520 \mathrm{mc}$ langen Pause vor der Wiederholung von das mögt ihr alles falsch finden endet. Eine besondere Intensität des Ausrufs im Schlussteil seiner appellativen Welle wird durch die Steigerung der Anzahl von Kernteilen der Grundfrequenz erreicht, welche auf der Frequenz von $250-300 \mathrm{~Hz}$ konzentriert sind, während beim langsamen Start ihre Lokalisierung sich auf der $120-150 \mathrm{~Hz}$ Höhe befand. Außerdem wird die besondere Intensität durch die erhöhte Klangintensität erreicht; sie kennzeichnet sich durch die Amplitudenspanne von $60 \mathrm{~dB}$ beim Start bis $88 \mathrm{~dB}$ im Schlussteil der kleinen appellativen Welle an hauptbetonten Wörtern alles, welche durch ein aktives Kopfnicken verstärkt werden. Auf diese Weise erreicht J. Fischer in diesem Abschnitt des Appells vom Beginn bis zum Ende eine Frequenzspanne von $150 \mathrm{~Hz}$ und Lautstärkespanne von $30 \mathrm{~dB}$, was von dem Hörer als ein starker «Energieausbruch», oder «Charisma-Ausbruch» wahrgenommen wird.

Die prominenteste Vertreterin des politischen Diskurses der ukrainischen Frauen, Julia Timoschenko, verfügt über umfangreiche rhetorische Erfahrung in öffentlichen Auftritten und verwendet alle Mittel politischer Rhetorik, einschließlich Methoden der stimmlich-gestischen Beeinflussung. Die Prosodik von Julia Timoschenko charakterisiert sich durch 
hohe Schallstärke, Klangfülle und weist im Gegenteil zu Joschka Fischer keine abrupten Veränderungen im temporalen, tonalen und dynamischen Struktur im Laufe ihres ganzen Auftrittes auf. Obwohl die Amplitudenwerte von J. Timoschenko auch wie bei Fischer im höheren Lautstärkebereich zwischen 80 und $90 \mathrm{~dB}$ liegen, wird beim Abhören ihrer Reden ein monotoner Eindruck geschaffen: die Amplitudenspanne sehr gering und beträgt kurz einmal ein Dezibel, also zwischen 82 (Aufruf: Ich will euch fragen, meine Mitbrüder: ob wir ohne eine solche Widerstand durchhalten konnten?/ Я хочу запитати у вас, мої побратими: а чи можна було без такого протистояння? und $83 \mathrm{~dB}$ (Und darum - hebt den Kopf hoch! Nehmt die Haltung an! / I тому - підніміть голови, розправте плечі!). Eine solche Monotonität, solch ein Klagelied, ist für alle Auftritte von Julia Timoschenko charakteristisch, was einerseits von einem hohen Grad der Emotionsspannung und anderseits von einem starken Charakter und einem kämpferischen Willen zeugt.

Das kinetische Werkzeugset, das an der Gestaltung und Stärkung der wichtigsten Orte in öffentlichen Reden von Julia Timoschenko beteiligt ist, steht im Zusammenhang mit dem aktiven Gebrauch von Co-Speech-Gesten wie (1) synchrones Kopfnicken, (2) redebegleitende Gesten von beiden Händen mit einer faustartigen Handfläche, (3) eine offene Handfläche mit Zeigefinger, der auf das Publikum gerichtet ist, sowie eine bestimmte Geste (4), wenn sich die Spitzen aller fünf Finger zu einer Prise zusammenfalten und die Hand in dieser Konfiguration des Pinsels sich in der Richtung des Publikums bewegt.

So kommen wir zur Notwendigkeit, ein integratives Modell des inspirierenden Diskurses eines charismatischen Politikers in beiden Sprachen aufzu- bauen, in dem sich die Begeisterung an den prominenten Stellen durch entsprechende verbale - kurze Imperativsätze, prosodische - Amplitude- und Tonhöhensprünge und kinetische Mittel - redebegleitende Bewegung der rechten oder linken Hand isomorph zum Ausdruck gebracht werden kann. Es ist wichtig dabei zu berücksichtigen, dass sich paraverbale Kontraste in den charismatischen Diskursen der ukrainischen und deutschen Politiker vor allem im rhapsodischen Aufbau der Prosodie für deutsche Politiker, und im monotonen - für ukrainische zeigen.

Was die weiteren Perspektiven der Charisma-Idealtyps angeht, ist sowohl in Deutschland als auch in der Ukraine die Suche nach zukünftigen Charismatikern ausgehend von der Initiative der Massen, bzw. der spontanen Bewegung «von unten» ${ }^{14}$, den sogenannten «Graswurzeln» (engl. grassroots) von entscheidender Bedeutung. Das «Grassroots-Charisma» der potenziellen Hoffnungsträger befindet sich erst im Anfangsstadium, also im Keim. Es wird ihnen noch nicht zugeschrieben und wird nicht in den Medien manifestiert aber seine Merkmale sind bei Nachwuchspolitikern zu finden, wenn sie bei Kundgebungen, Streikposten, Unterschriftensammeln und anderen sozialen und politischen Aktionen direkt beobachtet werden. Wichtig ist hier der Moment der ersten öffentlichen Zuschreibung von Charisma in den Medien und sozialen Netzwerken von Anhängern und Gegnern zugleich sowie ihre aktive Kampfstellung und Opposition zu den alten Parteistrukturen und insbesondere zu ihren nicht charismatischen und nicht leidenschaftlichen Parteichefs (vgl. Rede von Kevin Kühnert, Bundesvorsitzender Jusos, beim SPD-Bundesparteitag zur Aufnahme von Koalitionsgesprächen in Bonn, 21.01.2018 ${ }^{15}$ ).

\section{LITERATURVERZEICHNIS}

Петлюченко Н. B. Geburt und Tod des Charismas: Maidan 2004 vs. Euromaidan 2014 (diskursive Besonderheiten der Appelle in der Antrittsrede von Petro Porošenko bei seiner Amtseinführung am 7. Juni 2014)/ Н.В.Петлюченко // Одеський лінгвістичний вісник : зб. наук. праць; Національний університет «Одеська юридична академія». - Вип. 4.- Одеса : «Видавничий дім «Гельветика», 2014. - С. 336-341.

Петлюченко Н. В. Grassroots-Charisma: diskursive und experimentell-phonetische Identifikation potenzieller Charismatiker in der Graswurzelbewegung Deutschlands und der Ukraine / Н.В.Петлюченко // Одеський лінгвістичний вісник : зб. наук. праць; Національний університет «Одеська юридична академія». - Вип. 11.- Одеса : «Видавничий дім «Гельветика», 2018. - С. $159-166$.

Петлюченко Н. В. Харизматика : мовна особистість і дискурс : монографія / Н. В. Петлюченко. - Одеса : Астропринт, 2009. $-464 \mathrm{c}$.

Dempsey J. Das Phänomen Merkel: Deutschlands Macht und Möglichkeiten / Judy Dempsey. - Hamburg : Edition Körber-Stiftung, 2013. - 208 S.

Encke J. Charisma und Politik: Warum unsere Demokratie mehr Leidenschaft braucht / Julia Encke. - München : Hanser Verlag, 2014. - $176 \mathrm{~S}$.

Haese I. Stadt und Charisma : Eine akteurszentrierte Studie in Zeiten der Schrumpfung / Inga Haese. - Wiesbaden : Springer Fachmedien Wiesbaden, 2017. - $252 \mathrm{~S}$.

Herbst L. Hitlers Charisma: die Erfindung eines deutschen Messias / Ludolf Herbst. - Frankfurt am Main: Fischer-TaschenbuchVerl., 2011. - $330 \mathrm{~S}$.

Kühnert, K. Y. Rede von Kevin Kühnert, Bundesvorsitzender Jusos, beim SPD-Bundesparteitag zur Aufnahme von Koalitionsgesprächen in Bonn, 21.01.2018. https://www.youtube.com/watch?v=VPffCJj6y9g

\footnotetext{
${ }^{14}$ Petliuchenko 2018

${ }^{15}$ Kühnert 2018
} 
Langguth G. Kohl, Schröder, Merkel: Machtmenschen / Gerd Langguth. - München : Deutscher Taschenbuch Verlag, 2009. - 580 S.

Petljutschenko N. Grassroots-Charisma: diskursive und experimentell-phonetische Identifikation potenzieller Charismatiker in der Graswurzelbewegung Deutschlands und der Ukraine / Н.В.Петлюченко // Одеський лінгвістичний вісник : зб. наук. праць / [гол. ред. Н. В. Петлюченко] ; Національний університет «Одеська юридична академія». - Вип. 11. Одеса : «Видавничий дім «Гельветика», 2018. - C. 159-166. (DOI 10.32837/2312-3192-2018-11-159-166).

Petlyuchenko N. Aggressive Rhetoric: Prosodic and Kinetic Means / Nataliya Petlyuchenko, Anna Artiukhova // Proceedings of International Conference «Gesture and Speech in Interaction» (GESPIN). - Nantes, France, September 2-4, 2015. - P. $191-194$.

Schmölders C. Hitlers Gesicht: eine physiognomische Biographie / Claudia Schmölders. - München : Beck, 2000. - 264 S.

Schwinn Th. Alte Begriffe - neue Probleme : Max Webers Soziologie im Lichte aktueller Problemstellungen / Thomas Schwinn (Hrsg.). - Tübingen : Mohr Siebeck, 2016. - 465 S.

Weber M. Wirtschaft und Gesellschaft. Grundriss der verstehenden Soziologie. - Paderborn: Voltmedia, 2006. - 1311 S.

\section{REFERENCES}

Dempsey, J. (2013). Das Phänomen Merkel: Deutschlands Macht und Möglichkeiten. Hamburg: Edition Körber-Stiftung. Encke, J. (2014). Charisma und Politik: Warum unsere Demokratie mehr Leidenschaft braucht. München : Hanser Verlag. Gössler, S. (2009). Barack Obama: seine Sprache - seine Stärke - sein Charisma; Rhetorik einer Erfolgsgeschichte. Norderstedt: Books on Demand.

Haese, I. (2017). Stadt und Charisma : Eine akteurszentrierte Studie in Zeiten der Schrumpfung. Wiesbaden: Springer Fachmedien Wiesbaden.

Herbst, L. (2011). Hitlers Charisma: die Erfindung eines deutschen Messias. Frankfurt am Main: Fischer-Taschenbuch-Verl.

Kühnert, K. Y. (2018). Rede von Kevin Kühnert, Bundesvorsitzender Jusos, beim SPD-Bundesparteitag zur Aufnahme von Koalitionsgesprächen in Bonn, 21.01.2018 [Speech by Kevin Kühnert, Federal Chairman Jusos, at the SPD Federal Party Congress on Coalition Talks in Bonn, 21.01.2018]. https://www.youtube.com/watch?v=VPffCJj6y9g

Langguth, G. (2009). Kohl, Schröder, Merkel: Machtmenschen. München: Deutscher Taschenbuch Verlag.

Petliuchenko, N. V. (2009). Charyzmatyka : movna osobystist' i dyskurs [Харизматика : мовна особистість і дискурс]. Odessa: Astroprint.

Petliuchenko, N. (2018). Grassroots-Charisma: diskursive und experimentell-phonetische Identifikation potenzieller Charismatiker in der Graswurzelbewegung Deutschlands und der Ukraine. Odeskyi linhvistychnyi visnyk [Одеський лінгвістичний вісник] 11, pp. 159-166 (DOI 10.32837/2312-3192-2018-11-159-166)

Petljutschenko, N. (2014). Geburt und Tod des Charismas: Maidan 2004 vs. Euromaidan 2014 (diskursive Besonderheiten der Appelle in der Antrittsrede von Petro Porošenko bei seiner Amtseinführung am 7. Juni 2014). Odeskyi linhvistychnyi visnyk [Одеський лінгвістичний вісник] 4, pp. 336-341.

Petlyuchenko N., and Artiukhova, A. (2015). Aggressive Rhetoric: Prosodic and Kinetic Means. In: Proceedings of International

Conference «Gesture and Speech in Interaction» (GESPIN) 2015, Nantes, France, pp. 191-194.

Schmölders, C. (2000). Hitlers Gesicht: eine physiognomische Biographie. München: Beck.

Schwinn, Th. ed. (2016). Alte Begriffe - neue Probleme : Max Webers Soziologie im Lichte aktueller Problemstellungen. Tübingen : Mohr Siebeck.

Weber, M. (2006). Wirtschaft und Gesellschaft. Grundriss der verstehenden Soziologie. Paderborn: Voltmedia, 2006.

\section{Анотація}

У статті представлено аналіз мовленнєвих корелятів харизми політичних лідерів у сучасному німецькому та українському політичному дискурсі і робиться спроба надати відповідь на питання, чи може ідеальний тип бути об'єктом мультимодальних фонетичних досліджень. Дискурсивний опис харизматичних лідерів у німецькому та українському політичному дискурсах та визначення їх контрастних ознак спирається на біологічні, соціальні, психологічні та лінгвістичні параметри, що формують їх харизматичні портрети. Вирішальне значення в цьому контексті має риторика публічного виступу, виразна бійцівська позиція, ентузіазм, наснага політика, які відбиваються у просодичних та кінетико-мімічних характеристиках мовця та сприймаються і приписуються йому/їі як харизма.

Просодична специфіка харизматичного дискурсу характеризується посиленням усіх його динамічних, тональних і темпоральних компонентів. У фонетичних дослідженнях такий акустичний ефект називається просодичним виділенням або просодичною емфазою. У даній роботі використовується термін просодична інтенсивність, під якою розуміється різка зміна висоти, гучності, темпу і пауз на змістовно навантажених ділянках мовлення, де містяться звернення, заклики, концепти із ціннісною семантикою. Харизматична риторика формується також кінетичною (кінетико-мімічною) складовою, яка функціонально пов'язана з просодичним оформленням мови і робить вплив більш ефективним. Харизматичні політичні меседжі супроводжуються акцентуючими co-speech-жестами одної або двох рук, кивками голови та рухами усього тіла, які підкреслюють ключові моменти всього виступу, тім самим підсилюючи загальний вплив усього виступу. Жест сприймається адресатом як «кінематична» форма вербальної харизматичної апеляції, через яку політик здійснює свій вплив на своїх послідовників та/або опонентів, заохочуючи їх до вчинення сумісних дій, спрямованих на досягнення певної мети. Харизматична риторика зароджується в політичній комунікації в умовах кризи або передвиборчій боротьбі і притаманна тим політикам, чия позиція не відповідає позиції більшості. Харизматичний ентузіазм і натхнення мають свою етноспецифіку (німецька рапсодичність, українська монотонність) і відображені в просодичному і жестовому виділенні, яке супроводжує як самостійні, так і службові або окремі частини мови і має автономність як модель харизматичної риторики. Результати цього дослідження можуть бути застосовані в дискурсивних студіях, прикладній фонетиці, порівняльній лінгвістиці та політичній комунікації.

\section{Ключові слова}

Ідеальний тип, харизма, просодична інтенсивність, ентузіазм, натхнення, рапсодичність, монотонність, co-speеch-жести, міміка. 